\title{
Effect of Portal Hypertension in the Small Bowel: An Endoscopic Approach
}

\author{
Pedro Figueiredo $\cdot$ Nuno Almeida $\cdot$ Clotilde Lérias · Sandra Lopes $\cdot$ \\ Hermano Gouveia $\cdot$ Maximino C. Leitão $\cdot$ Diniz Freitas
}

Received: 29 June 2007 / Accepted: 27 October 2007/Published online: 17 November 2007

(C) Springer Science+Business Media, LLC 2007

\begin{abstract}
Background and aim The effects of portal hypertension in the small bowel are largely unknown. The aim of the study was to prospectively assess portal hypertension manifestations in the small bowel. Methods We compared, by performing enteroscopy with capsule endoscopy, the endoscopic findings of 36 patients with portal hypertension, 25 cirrhotic and 11 non-cirrhotic, with 30 controls. Results Varices, defined as distended, tortuous, or saccular veins, and areas of mucosa with a reticulate pattern were significantly more frequent in patients with PTH. These two findings were detected in 26 of the 66 patients (39\%), 25 from the group with PTH (69\%) and one from the control group $(3 \%)(P<0.0001)$. Among the 25 patients with PTH exhibiting these patterns, 17 were cirrhotic and 8 were non-cirrhotic $(P=0.551)$. The presence of these endoscopic changes was not related to age, gender, presence of cirrhosis, esophageal or gastric varices, portal hypertensive gastropathy, portal hypertensive colopathy, prior esophageal endoscopic treatment, current administration of beta-blockers, or Child-Pugh Class C. More patients with these endoscopic patterns had a previous history of acute digestive bleeding ( $72 \%$ vs. $36 \%$ ) $(P=0.05)$. Active bleeding was found in two patients (5.5\%). Conclusions The presence of varices or areas of mucosa with a reticulate pattern are manifestations of portal hypertension in the small bowel, found in both cirrhotic and non-cirrhotic patients. The clinical implications of these findings, as regards digestive bleeding, are
\end{abstract}

P. Figueiredo $(\varangle) \cdot$ N. Almeida · C. Lérias · S. Lopes ·

H. Gouveia · M. C. Leitão · D. Freitas

Department of Gastroenterology, Hospitais da Universidade de Coimbra, Av. Bissaya Barreto, Coimbra 3000-075, Portugal

e-mail: pedro.n.figueiredo@clix.pt uncertain, although we documented acute bleeding from the small bowel in two patients $(5.5 \%)$.

Keywords Capsule endoscopy - Portal hypertension · Small bowel

\section{Introduction}

One consequence of portal hypertension (PTH) is bleeding, particularly from esophageal varices [1]. However, other sources of digestive bleeding have been identified, including gastric [2] and colorectal mucosa [3, 4].

Portal hypertensive gastropathy (PHG) is a well-established cause of bleeding in cirrhotic patients, present in up to $98 \%$ of patients with PTH [5] and accounting for $25.8 \%$ of acute bleeding episodes in a cohort of 315 followed over a mean period of 18 months [2]. Changes in the gastric mucosa of patients with PTH were first described by McCormack et al. [6], but the mechanisms implicated in the pathogenesis of PHG are not yet fully understood. Portal hypertension, and not cirrhosis, is the main factor, because PHG has been described in patients with and without liver disease [7]. There is, however, no good correlation between the endoscopic findings and portal pressure [8], and gastric blood flow studies showed both increased and reduced gastric perfusion $[9,10]$.

Similarly, endoscopic abnormalities in the large bowel of patients with cirrhosis have also been described [3, 4, 11-14]. The spectrum of lesions categorized as portal hypertensive colopathy (PHC) includes findings resembling colitis (edema, diffuse hyperemia, friability, granularity, and/or spontaneous bleeding) and vascular lesions (cherry red spots, telangiectasias, or angiodysplasia-like lesions) [11], although colonic and ano-rectal 
varices can also be found in up to 31 and $38 \%$, respectively, of cirrhotic patients [3, 4]. As in PHG, these lesions are not solely detected in the presence of cirrhosis, but can also be found in cases of extra-hepatic portal vein obstruction [4, 12]. Portal hypertensive colopathy seems to be a rare cause of bleeding [3, 4].

The recognized existence of PHG and PHC suggests that the small bowel might also show endoscopic changes related to PTH. In fact, in 1989, Thiruvengadam and Gostout reported three patients, presenting with blood loss, who had diffuse erythema and scattered petechia in the stomach, but also in the duodenum and jejunum [15]. The small bowel, previously considered to be the most difficult segment of the gut to study, is nowadays easily explored with the advent of new endoscopic methods, for example capsule endoscopy (CE) and double-balloon enteroscopy $[16,17]$. The aim of this study was to assess PTH manifestations in the small bowel of cirrhotic and non-cirrhotic patients by performing enteroscopy with $\mathrm{CE}$.

\section{Patients and methods}

The cohort consisted of patients with PTH, documented by endoscopic evidence of esophageal or gastric varices, and included both cirrhotic and non-cirrhotic cases (the presence of cirrhosis was confirmed by histology, or by compatible physical, laboratory, and ultrasound studies). The control group included patients submitted to $\mathrm{CE}$, previously assessed by upper endoscopy, colonoscopy, abdominal ultrasound, liver biochemistry, and prothrombin time, that showed no evidence of PTH. Age less than 18 years, pregnancy, a prior history of bowel obstruction, major abdominal surgery, or any mental condition precluding compliance with the study were considered exclusion criteria.

Cirrhosis severity was graded according to the ChildPugh score [18] and esophageal varices using criteria proposed by Conn [19]. Portal hypertensive gastropathy, if present, was classified after McCormack et al. [6]. Portal hypertensive colopathy was defined by the presence of diffuse hyperemia and edema resembling chronic colitis, spontaneous bleeding, cherry red spots, angiodysplasia-like lesions, telangiectasias, and varices.

Informed consent was obtained from all patients. Data collected on each patient included age, sex, etiology of liver disease, Child-Pugh class, history of previous acute digestive bleeding, findings of upper digestive endoscopy and colonoscopy, endoscopic sclerotherapy or band ligation for eradication of esophageal varices, and treatment with beta-blockers.

A PillCam SB (Given Imaging, Yoqneam, Israel) was used. The patients fasted for $12 \mathrm{~h}$ before the examination and drank a solution that contained simethicone immediately before swallowing the capsule. All the patients were advised to drink after $4 \mathrm{~h}$ and, after $8 \mathrm{~h}$, the sensor array and the recording device were removed. The digital video image streams of the examinations were downloaded to the RAPID system.

Statistical comparisons were made using the chi-squared test, with the Yates correction when needed, the Fisher exact test, and the Student $t$-test. A $P$-value of less than 0.05 was considered statistically significant. Statistical analysis was performed with statistical software (SPSS version 11,5, SPSS, Chicago, Illinois, USA).

\section{Results}

Between November 2002 and February 2006, 66 patients, 36 with PTH and 30 controls, were enrolled on the study.

The most common etiology of PTH was alcoholic cirrhosis and, in non-cirrhotic patients, portal vein thrombosis (Table 1). Among the eleven patients without cirrhosis, nine had been previously submitted to hepatic biopsy that confirmed the absence of changes in the hepatic parenchyma. The mean age was $56 \pm 12$ years (range 33-81), and the dominant gender was male $(83 \%)$. All the patients presented esophageal or gastric varices. Twenty-two $(61 \%)$ had a previous history of acute digestive bleeding, and 21 $(58 \%)$ received sclerotherapy or band ligation. Twelve patients $(33 \%)$ were on therapy with beta-blockers. Portal hypertensive gastropathy was present in 27 (75\%) of the patients. Among the 22 patients submitted to colonoscopy, PHC was diagnosed in six (27\%). A Child-Pugh score was attributed to all cirrhotic patients; Class B was the most common, found in 16 (64\%) patients, followed by Class C, detected in six (24\%) patients then class A detected in three (12\%). The demographic, clinical, and endoscopic characteristics of cirrhotic and non-cirrhotic patients with PTH are presented in Table 2.

Table 1 Portal hypertension etiology

\begin{tabular}{lc}
\hline Portal hypertension etiology & Number $(\%)$ \\
\hline Cirrhotic & $25(69.4)$ \\
Alcohol & $23(63.8)$ \\
Virus & $1(2.8)$ \\
Primary biliary cirrhosis & $1(2.8)$ \\
Non-cirrhotic & $11(30.6)$ \\
Portal vein thrombosis & $8(22.2)$ \\
Budd-Chiari syndrome & $1(2.8)$ \\
Unknown & $2(5.6)$ \\
Total & $36(100)$ \\
\hline
\end{tabular}


Table 2 Demographic, clinical, and endoscopic findings of patients with PTH

${ }^{\text {a }}$ Only 22 patients with PTH were submitted to colonoscopy

\begin{tabular}{llll}
\hline & $\begin{array}{l}\text { Cirrhotic patients } \\
(N=25)\end{array}$ & $\begin{array}{l}\text { Non-cirrhotic patients } \\
(N=11)\end{array}$ & $P$ \\
\hline Mean age (years) & $59 \pm 10$ & $49 \pm 15$ & 0.02 \\
Gender (male/female) (\%) & $88 / 12$ & $73 / 27$ & 0.25 \\
Previous acute digestive bleeding, $n(\%)$ & $14(56)$ & $8(73)$ & 0.28 \\
Prior esophageal sclerotherapy/ligation, $n(\%)$ & $13(52)$ & $8(73)$ & 0.21 \\
Prior beta-adrenergic blockers, $n(\%)$ & $8(32)$ & $4(36)$ & 0.54 \\
Esophageal varices, $n(\%)$ & $23(92)$ & $8(73)$ & 0.15 \\
Gastric varices, $n(\%)$ & $2(8)$ & $3(27)$ & 0.15 \\
Portal hypertensive gastropathy, $n(\%)$ & $22(88)$ & $5(46)$ & 0.01 \\
Portal hypertensive colopathy ${ }^{\mathrm{a}}, n(\%)$ & $4 / 17(24)$ & $2 / 5(40)$ & 0.58 \\
\hline
\end{tabular}

Table 3 Endoscopic findings in the small bowel

The control group consisted of thirty patients, age and gender-matched, consecutively admitted to our outpatient clinic to undergo $\mathrm{CE}$ due to obscure gastrointestinal bleeding $(n=21)$, suspected inflammatory bowel disease $(n=7)$, and abdominal pain $(n=2)$.

The colon was reached during the recording time in 55 patients $(83.3 \%)$ and the ileum in the remaining 11 patients. No technical problems or complications occurred during the examinations, and the capsule was naturally excreted by all patients.

The endoscopic findings in patients with PTH and in the control group are shown in Table 3. Varices, defined as distended, tortuous, or saccular veins (Fig. 1), and areas of mucosa with a reticulate pattern (Fig. 2) were significantly more frequent in patients with PTH. These two findings were detected in 26 of the 66 patients (39\%), 25 from the group with PTH (69\%) and one from the control group $(3 \%)(P<0.0001)$ (Table 4). Among the 25 patients with PTH exhibiting these patterns, seventeen were cirrhotic and eight were non-cirrhotic $(P=0.551)$ (Table 4$)$. Two cirrhotic patients presented both patterns, while 15 presented only enteropathy. In the non-cirrhotic group, five presented the two patterns and three exhibited only varices. No

\begin{tabular}{lllc}
\hline & $\begin{array}{l}\text { Patients } \\
\text { with PTH, } N(\%)\end{array}$ & $\begin{array}{l}\text { Control } \\
\text { group, } N(\%)\end{array}$ & $P$ \\
\hline $\begin{array}{l}\text { Varices } \\
\text { Reticulate pattern }\end{array}$ & $10(27.8)$ & $1(3.3)$ & 0.008 \\
$\quad$ of the mucosa & $22(61.1)$ & $1(3.3)$ & $<0.0001$ \\
$\begin{array}{l}\text { Angiodysplasia } \\
\text { Ulcerations or erosions }\end{array}$ & $10(27.8)$ & $12(49)$ & 0.216 \\
\hline
\end{tabular}

statistically significant association was found between the two endoscopic patterns considered.

Active bleeding was detected in two patients (5.5\%) and one of these was submitted to double-balloon enteroscopy. The procedure confirmed the presence of areas of congestive mucosa with a reticulate pattern (Fig. 3), but the site of bleeding was one small angiodysplasia located in the jejunum, successfully treated with argon-plasma.

Grouping patients with PTH according to the presence of these two endoscopic patterns in the small bowel (Table 5) showed there were no statistically significant differences with regard to age, gender, presence of cirrhosis, esophageal or gastric varices, PHG, PHC, prior
Fig. 1 Areas of mucosa with a reticulate pattern
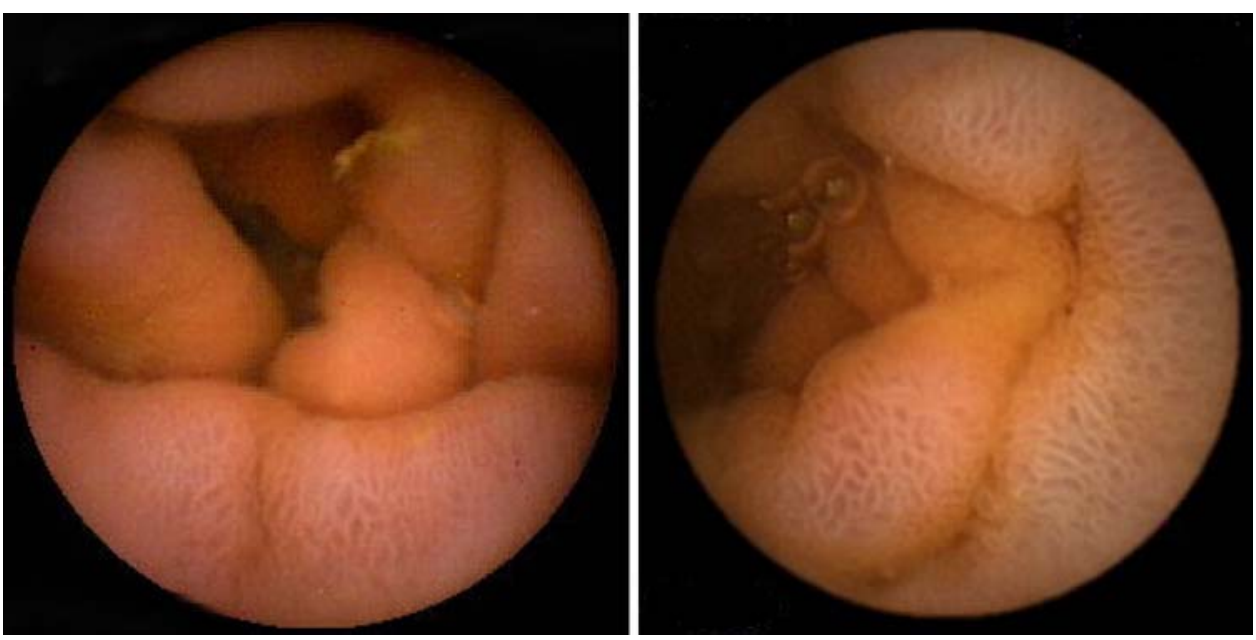
Fig. 2 Small-bowel varices
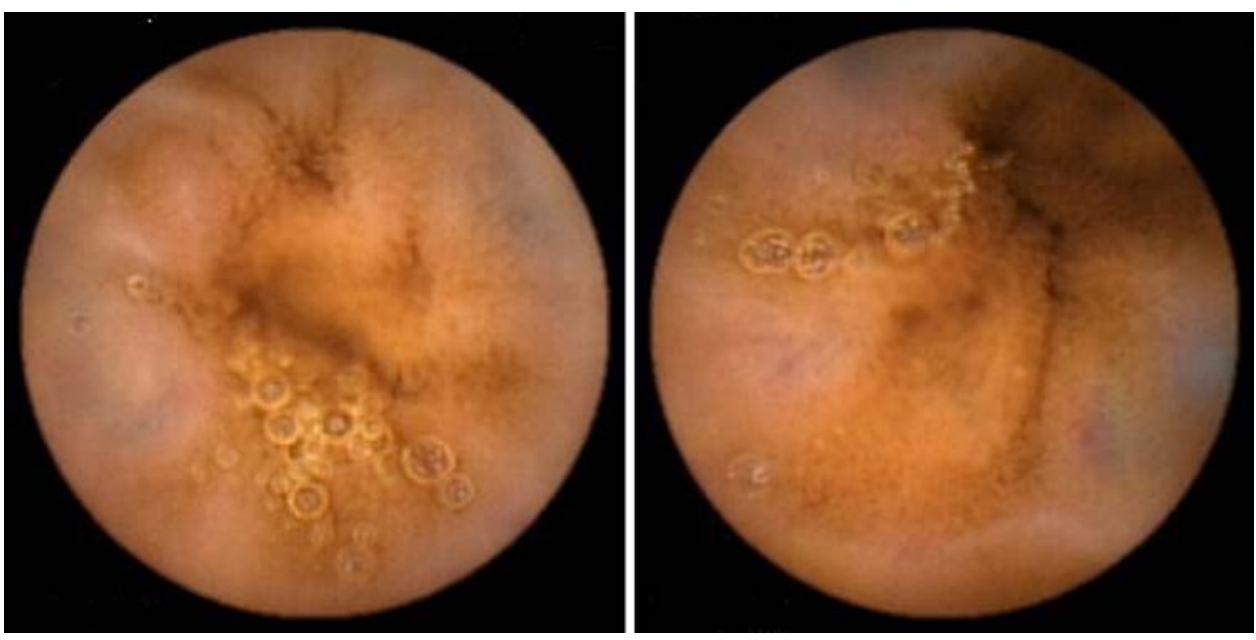

Table 4 Number of patients showing varices or a reticulate pattern of the mucosa

\begin{tabular}{llc}
\hline & Patients $(N)$ & $\begin{array}{c}\text { Varices/reticulate } \\
\text { pattern, } N(\%)\end{array}$ \\
\hline Cirrhotic PTH & 25 & $17(68)$ \\
Non-cirrhotic PTH & 11 & $8(73)$ \\
Controls & 30 & $1(3)$ \\
Total & 66 & $26(39)$ \\
\hline
\end{tabular}

esophageal endoscopic treatment, current administration of beta-blockers, or Child-Pugh Class C. More patients from the group with PTH manifestations in the small bowel had a previous history of acute digestive bleeding (72\% vs. $36 \%)(P=0.05)$. The difference did not acquire statistical significance when the two endoscopic patterns were considered separately.

Other lesions found in patients with PTH included small polyps and parasites in two, one case of inflammatory stricture, and one of submucosal tumor. In the control group, one case of villous atrophy and one case of a submucosal tumor were also detected.

\section{Discussion}

The consequences of PTH in the digestive tract are easily studied in the segments that are accessible to flexible endoscopy. In fact, McCormack et al. [6], in 1985, were the first to describe the entity called congestive gastropathy, but similar changes were later reported to occur also in the duodenum [15, 20, 21] and large bowel [3, 4, 11-14].

Congestive gastroenteropathy has been reported to be a non-variceal cause of bleeding in patients with PTH [15, 20], but most of the studies on portal hypertensive enteropathy (PHE) found in the literature were obtained by upper endoscopy [15, 20], by push enteroscopy [22], or by retrograde ileoscopy at colonoscopy [23, 24], and only include the duodenum, proximal jejunum, and terminal ileum.

Because of its distance from the mouth and anus, the small bowel has, for many years, been considered the most difficult segment of the gut to study with flexible endoscopy. With the advent of CE [16], it is now possible to examine the entire small intestine without discomfort to the patient and on an
Fig. 3 Areas of congestive mucosa with a reticulate pattern:

(a) capsule endoscopy; (b) double-balloon enteroscopy
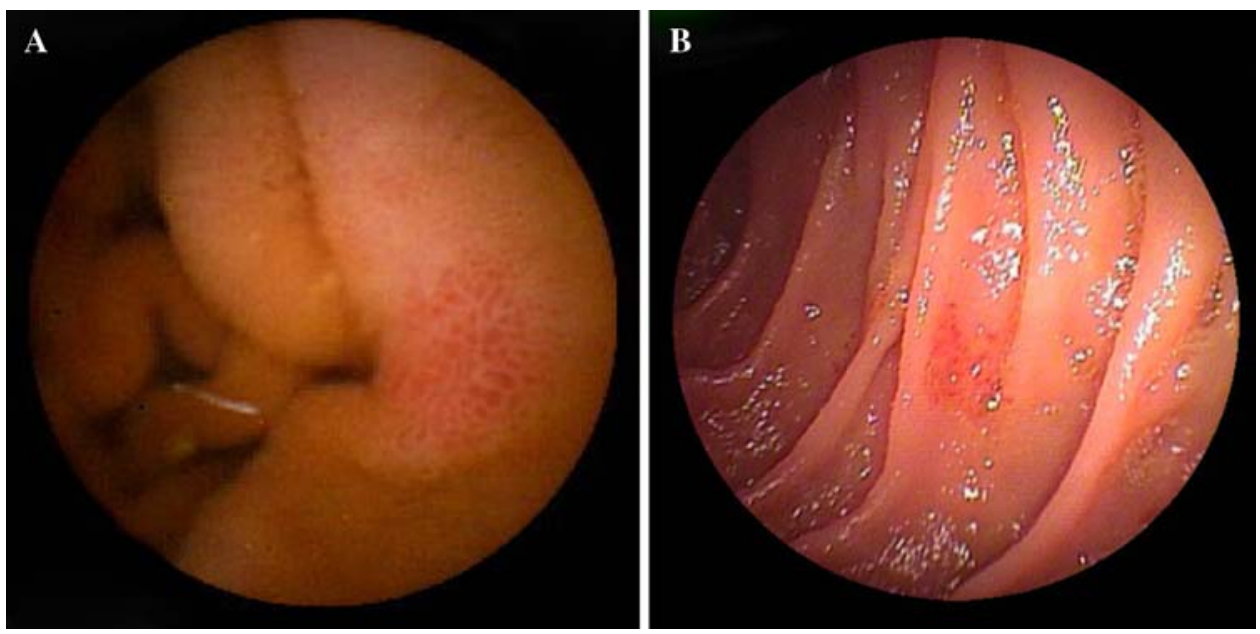
Table 5 Comparison of the patients with PTH according to the presence of varices or a reticulate pattern of the mucosa in the small bowel

\begin{tabular}{llll}
\hline & $\begin{array}{l}\text { Findings present } \\
(N=25)\end{array}$ & $\begin{array}{l}\text { Findings absent } \\
(N=11)\end{array}$ & $P$ \\
\hline Mean age (years) & $57 \pm 13$ & $53 \pm 11$ & 0.42 \\
Male gender (\%) & 80 & 91 & 0.39 \\
Cirrhosis, $n$ (\%) & $17(68)$ & $8(73)$ & 0.55 \\
Oesophageal varices, $n(\%)$ & $22(88)$ & $9(82)$ & 0.49 \\
Gastric varices, $n$ (\%) & $2(8)$ & $3(27)$ & 0.15 \\
Portal hypertensive gastropathy, $n(\%)$ & $18(72)$ & $9(82)$ & 0.42 \\
Portal hypertensive colopathy, $n(\%)$ & $4 / 17(24)$ & $2 / 5(40)$ & 0.46 \\
Previous acute digestive bleeding, $n(\%)$ & $18(72)$ & $4(36)$ & 0.05 \\
Prior esophageal sclerotherapy/ligation, $n(\%)$ & $17(68)$ & $4(36)$ & 0.08 \\
Prior beta-adrenergic blockers, $n(\%)$ & $8(32)$ & $4(36)$ & 0.54 \\
Child-Pugh class C, $n$ (\%) & $5(20)$ & $1(9)$ & 0.34 \\
\hline
\end{tabular}

the edge of statistical significance is previous acute digestive bleeding $(P=0.05)$. So, although the significance of this finding is uncertain, active bleeding from the small bowel was documented in two patients (5.5\%). This figure is not substantially different from that reported for PHG and PHC [2, 3].

Another important issue is whether angiodysplastic-like lesions, detected by $\mathrm{CE}$, should be considered a small bowel manifestation of PTH. Although angiodysplasia seems to be the main etiology of acute bleeding from the small bowel in patients with PTH, documented in all cases in the series presented by De Palma et al. [27] and in one of the two cases in our series, the frequency of detection of these lesions was, in our study, similar in patients with PTH and in the control group. Therefore, angiodysplasticlike lesions should not, in our opinion, be considered a small bowel manifestation of PTH. Future studies, aiming the recognition of the clinical impact of small-bowel endoscopic findings in these patients (as regards bleeding episodes or transfusion requirements, for example), must separately consider those who present angiodysplastic-like lesions.

Two diagnostic options exist when dealing with small bowel manifestations of PTH. The first is to perform enteroscopy only in patients who present obscure digestive bleeding. This was the method adopted by De Palma et al., who found active bleeding in four patients (10.8\%), the source being angiodysplastic-like lesions [27]. The other diagnostic option is to include enteroscopy in the work-up of all cirrhotic patients, irrespective of the presence of hemorrhage. In our series, the patients were not selected according to the presence of previous bleeding, but only according to the existence of documented PTH, and we found that two patients (5.5\%) presented active bleeding. Not surprisingly, in view of the selection criteria, fewer patients were actively bleeding in our series. Given these results, we agree with JimenezSaenz et al. [30] that enteroscopy should be reserved for 
patients who present obscure digestive bleeding. Capsule enteroscopy should be the preferred method firstly because, by allowing complete examination of the small bowel, it is obviously more efficient in detecting small bowel manifestations of PTH than other diagnostic modalities, for example retrograde ileoscopy [23, 24], and, second, accurate diagnosis may enable therapeutic intervention with double-balloon enteroscopy, as occurred in our patient.

The relationship between previous esophageal endoscopic treatment and the existence of PHG and PHC is discussed in the literature. Sarin et al. found that among 88 patients with PHG, 74\% developed this condition after variceal eradication [29]. The severity of the PHG also seems to increase after endoscopic variceal treatment, either by sclerotherapy or by band ligation [31]. On the other hand, it seems that esophageal variceal band ligation does not affect the incidence of anorectal varices or PHC [32]. As regards PHE, the results of De Palma et al. indicate an absence of association between previous endoscopic variceal therapy and the presence of PHE [27]. In our series, although patients submitted to prior esophageal endoscopic treatment exhibited small-bowel manifestations of PTH more frequently, the result was not statistically significant $(P=0.08)$.

The concept of portal hypertensive intestinal vasculopathy, which includes PHG, PHE, and PHC, was selected by Viggiano and Gostout to describe the effects of PTH in the gut [33]. The association between PHG and PHC is well documented by Bini et al. for a large series of cirrhotic patients [11]. Two studies concerning PHE address this issue: one, published as a full paper by De Palma et al., found that PHE is significantly more common in the presence PHG and PHC [27], while the other, a study presented recently as an abstract by Repici et al., found no association between PHE, documented by $\mathrm{CE}$, and the presence of PHG and PHC [34]. Our results do not show that PHG and PHC are more frequently found in patients with small-bowel manifestations of PTH.

In summary, these data provide evidence that the presence of varices and areas of mucosa with a reticulate pattern should be considered manifestations of PTH in the small bowel, and that these endoscopic findings are not exclusively found in cirrhotic patients but can also be detected in non-cirrhotic patients with PTH. The clinical implications of these findings, as regards digestive bleeding, are uncertain, although we documented acute bleeding from the small bowel in two patients (5.5\%). The putative existence of a portal hypertensive intestinal vasculopathy, which includes portal hypertensive gastropathy, enteropathy, and colopathy, is not suggested by our results.

\section{References}

1. Garcia-Tsao G (2002) Portal hypertension. Curr Opin Gastroenterol 18:351-359

2. Primignani M, Carpinelli L, Preatoni P et al (2000) Natural history of portal hypertensive gastropathy in patients with liver cirrhosis. The New Italian Endoscopic Club for the study and treatment of esophageal varices (NIEC). Gastroenterology 119:181-187

3. Bresci G, Parisi G, Capria A (2006) Clinical relevance of colonic lesions in cirrhotic patients with portal hypertension. Endoscopy 38:830-835

4. Misra SP, Dwivedi M, Misra V et al (2005) Colonic changes in patients with cirrhosis and in patients with extrahepatic portal vein obstruction. Endoscopy 37:454-459

5. Burak KW, Beck PL (2003) Diagnosis of portal hypertensive gastropathy. Curr Opin Gastroenterol 19:477-482

6. McCormack TT, Sims J, Eyre-Brook I, Kennedy H, Goepel J, Johnson AG, Triger DR (1985) Gastric lesions in portal hypertension: inflammatory gastritis or congestive gastropathy? Gut 26:1226-1232

7. Bayraktar Y, Balkanci F, Uzunalimoglu B et al (1996) Is portal hypertension due to liver cirrhosis a major factor in the development of portal hypertensive gastropathy? Am J Gastroenterol 91:554-558

8. Kamath PS, Lacerda M, Ahlquist DA et al (2000) Gastric mucosal responses to intrahepatic portosystemic shunting in patients with cirrhosis. Gastroenterology 118:905-911

9. Gupta R, Sawant P, Parameshwar RV et al (1998) Gastric mucosal blood flow and hepatic perfusion index in patients with portal hypertensive gastropathy. J Gastroenterol Hepatol 13:921-926

10. Panes J, Bordas JM, Pique JM et al (1992) Increased gastric mucosal perfusion in cirrhotic patients with portal hypertensive gastropathy. Gastroenterology 103:1875-1882

11. Bini EJ, Lascarides CE, Micale PL et al (2000) Mucosal abnormalities of the colon in patients with portal hypertension: an endoscopic study. Gastrointest Endosc 52:511-516

12. Ganguly S, Sarin SK, Bhatia V et al (1995) The prevalence and spectrum of colonic lesions in patients with cirrhotic and noncirrhotic portal hypertension. Hepatology 21:1226-1231

13. Kozarek RA, Botoman VA, Bredfeldt JE et al (1991) Portal colopathy: prospective study of colonoscopy in patients with portal hypertension. Gastroenterology 101:1192-1197

14. Rabinovitz M, Schade RR, Dindzans VJ et al (1990) Colonic disease in cirrhosis. An endoscopic evaluation in 412 patients. Gastroenterology 99:195-199

15. Thiruvengadam R, Gostout CJ (1989) Congestive gastroenteropathy-an extension of nonvariceal upper gastrointestinal bleeding in portal hypertension. Gastrointest Endosc 35:504-507

16. Iddan G, Meron G, Glukhovsky A et al (2000) Wireless capsule endoscopy. Nature 405:417

17. Yamamoto H, Yano T, Kita $\mathrm{H}$ et al (2003) New system of double-balloon enteroscopy for diagnosis and treatment of small intestinal disorders. Gastroenterology 125:1556

18. Pugh RN, Murray-Lyon IM, Dawson JL, Pietroni MC, Williams $\mathrm{R}$ (1973) Transection of the oesophagus for bleeding oesophageal varices. Br J Surg 60:646-649

19. Conn HO (1967) Ammonia tolerance in the diagnosis of esophageal varices. A comparison of endoscopic, radiologic, and biochemical techniques. J Lab Clin Med 70:442-451

20. Nagral AS, Joshi AS, Bhatia SJ, Abraham P, Mistry FP, Vora IM (1993) Congestive jejunopathy in portal hypertension. Gut 34:694-697

21. Menchen L, Ripoll C, Marin-Jimenez I et al (2006) Prevalence of portal hypertensive duodenopathy in cirrhosis: clinical and haemodynamic features. Eur J Gastroenterol Hepatol 18:649-653 
22. Desai N, Desai D, Pethe V et al (2004) Portal hypertensive jejunopathy: a case control study. Indian J Gastroenterol 23:99-101

23. Misra SP, Dwivedi M, Misra V, Gupta M (2004) Ileal varices and portal hypertensive ileopathy in patients with cirrhosis and portal hypertension. Gastrointest Endosc 60:778-783

24. Rana SS, Bhasin DK, Jahagirdar S et al (2006) Is there ileopathy in portal hypertension? J Gastroenterol Hepatol 21:392-397

25. Rondonotti E, Herrerias JM, Pennazio M et al (2005) Complications, limitations, and failures of capsule endoscopy: a review of 733 cases. Gastrointest Endosc 62:712-716

26. Yamamoto H, Kita H, Sunada K et al (2004) Clinical outcomes of double-balloon endoscopy for the diagnosis and treatment of small-intestinal diseases. Clin Gastroenterol Hepatol 2:1010-1016

27. De Palma GD, Rega M, Masone S et al (2005) Mucosal abnormalities of the small bowel in patients with cirrhosis and portal hypertension: a capsule endoscopy study. Gastrointest Endosc 62:529-534

28. Gupta R, Saraswat VA, Kumar M et al (1996) Frequency and factors influencing portal hypertensive gastropathy and duodenopathy in cirrhotic portal hypertension. J Gastroenterol Hepatol 11:728-733
29. Sarin SK, Shahi HM, Jain M et al (2000) The natural history of portal hypertensive gastropathy: influence of variceal eradication. Am J Gastroenterol 95:2888-2893

30. Jimenez-Saenz M, Romero-Vazquez J, Caunedo-Alvarez A et al (2006) Capsule endoscopy: a useful tool in portal hypertensive enteropathy. Gastrointest Endosc 64:152

31. Yuksel O, Koklu S, Arhan M et al (2006) Effects of esophageal varice eradication on portal hypertensive gastropathy and fundal varices: a retrospective and comparative study. Dig Dis Sci 51:27-30

32. Misra SP, Misra V, Dwivedi M et al (2002) Effect of esophageal variceal band ligation on hemorrhoids, anorectal varices, and portal hypertensive colopathy. Endoscopy 34:195-198

33. Viggiano TR, Gostout CJ (1992) Portal hypertensive intestinal vasculopathy: a review of the clinical, endoscopic, and histopathologic features. Am J Gastroenterol 87:944-954

34. Repici A, Pennazio M, Ottobrelli A et al (2005) Endoscopic capsule in cirrhotic patients with portal hypertension: spectrum and prevalence of small bowel lesions. Endoscopy 37(Suppl 1):A72 